\title{
(8) $-3: 0$ \\ Visualisierung, ein hilfreiches Puzzleteil im Unterricht Drei Beispiele aus meinem Chemieunterricht
}

\section{Hans Ueli Ehrensperger ${ }^{\star}$}

${ }^{*}$ Correspondence: H.U. Ehrensperger, E-mail: h.ehrensperger@bluemail.ch, Am Stadtbach 7, CH-8500 Frauenfeld.

\section{Abstract: Es wird der Einsatz von eigenen Computerprogrammen im Chemieunterricht an Mittelschulen beschrieben.}

Keywords: Animationen · kovalente Bindung ·

Proteinbiosynthese $\cdot \mathrm{pH}-\mathrm{Skala} \cdot$ Titration

\section{Einleitung}

Beim Unterrichten im Fach Chemie sprechen wir von 'kleinsten Teilchen' und von 'Wechsel-wirkungen'. Wir sind aber eigentlich nie ganz sicher, ob die Vorstellung, die sich in unseren Köpfen geformt hat, auch wirklich in Übereinstimmung steht mit dem, was sich in den Köpfen der Lernenden heranbildet. Durch Interpretation von Experimenten, Diskussionen über Hypothesen erhält die Lehrkraft Einblick in die Bilder, die sich Schülerinnen und Schüler von der Materie zurechtlegen. In Ergänzung dazu stellen Zeichnungen, Simulationen und Animationen wertvolle Hilfsmittel dar. Im Laufe der Jahre sind an der Kantonsschule Frauenfeld einige Computerprogramme entstanden, welche den Praxistest bestanden haben. Auf einige Beispiele wird im Folgenden kurz hingewiesen.

\section{Ein Gefühl für die Logarithmusfunktion entwickeln}

Ohne ein Gefühl für die Logarithmusfunktion kann man viele Phänomene in der Säure/Base-Chemie nicht verstehen. Erste Versuche, die Zahlenverhältnisse einer $\mathrm{pH}$-Skala mit Spiralen darzustellen, misslangen. Mit der Animation 'pH-Spiel' sollte die Information visualisiert werden, dass sich $\mathrm{H}_{3} \mathrm{O}^{+}$- und $\mathrm{OH}^{-}$ Konzentrationen immer um einen Faktor 10 verändern, unabhängig davon, wo man sich auf der $\mathrm{pH}$-Skala um eine $\mathrm{pH}$-Einheit verschiebt (Abb. 1). Dass dies aber je nach Ort auf der pH-Skala mit dramatisch unterschiedlichem Stoffumsatz verbunden ist, stellte die Klasse mit Styropor-klötzen eindrücklich dar (Abb. 2). Der grosse Aufwand für eine $\mathrm{pH}-$ Änderung in den Randgebieten der pH-Skala und beim Einsatz eines Puffers wurde mit Hilfe der Animationen 'Titration' und 'Titration mit Puffer' emotional verdeutlicht. Dieses Gefühl wurde mit Hilfe der folgenden Aufgabe noch verstärkt: die pH-Skala-Darstellung (vgl. Abb. 2) war ergänzt durch je zwei kleine Bechergläser; eines war beschriftet mit $\mathrm{H}_{3} \mathrm{O}^{+}$ und enthielt eine bestimmte Menge Sand, entsprechend der $\mathrm{H}_{3} \mathrm{O}^{+}$ Konzentration; das andere mit $\mathrm{OH}^{-}$und enthielt eine bestimmte Menge Mehl, entsprechend der $\mathrm{OH}^{-}$-Konzentration (die Gläser im mittleren Bereich der $\mathrm{pH}-\mathrm{Skala}$ waren somit praktisch leer, im Rand-gebiet recht gefüllt). Jeder Schülerin und jedem Schüler wurde ein $\mathrm{pH}-$ Wert zugewiesen, verbunden mit der Aufgabe, mit Hilfe einer kleinen Pinzette die Mengen an Sand bzw. Mehl so $\mathrm{zu}$ verkleinern, dass die eigenen Bechergläser gleich aussehen, wie die Bechergläser der Schülerin zur Rechten bzw. zur Linken. Die Unmutsäusserungen bzw. die Schadenfreude - je nach pHWert - zeigten, dass die Klasse das Problem verinnerlicht hatte. Visualisierungen, die die Lehrkraft selbst kreiert, bieten den grossen Vorteil, dass ein einheitliches Darstellungsmuster die gewählte Intention verstärken kann. Selbstverständlich muss immer mit Experimenten und Klassendiskussionen (in diesem Fall etwa durch Titrationsexperimente mit Kurvendiskussionen) der Entstehung von Fehlbildern entgegengewirkt werden.

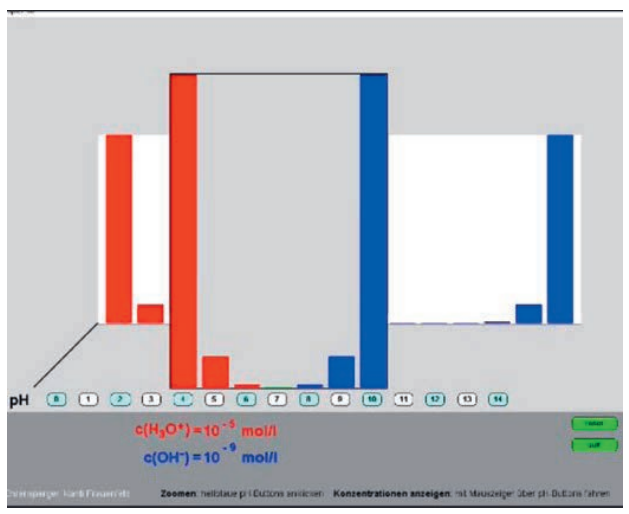

Abb. 1. Ausschnitt aus dem Programm 'pH-Spiel'.

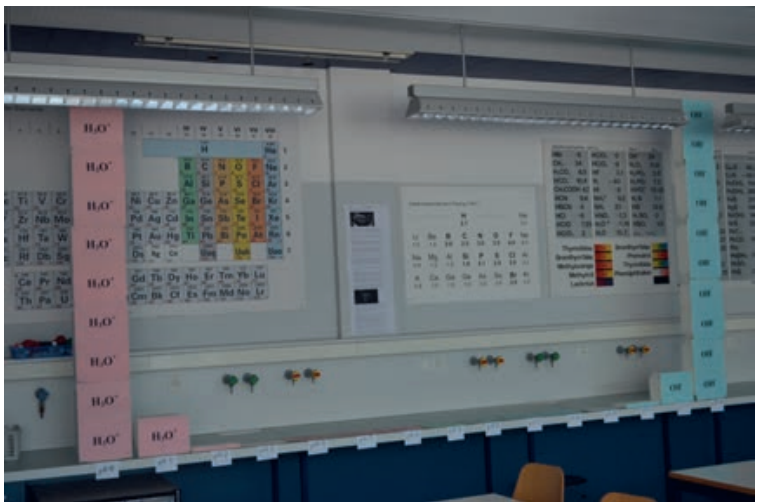

Abb. 2. Darstellung der $\mathrm{H}_{3} \mathrm{O}^{+}$- und $\mathrm{OH}^{-}-$Konzentrationen mit Styroporsäulen im Klassenzimmer.

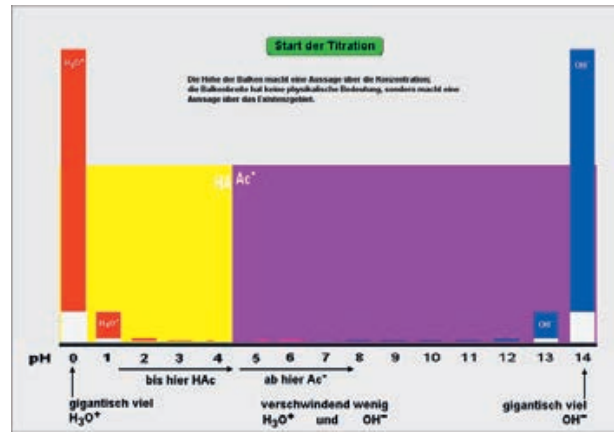

Abb. 3. Ausschnitt aus dem Programm 'Titration mit Puffer'. 


\section{Kovalente Bindung}

Eine Bindung als Wechselspiel von Anziehungs- und Abstossungskräften zu verstehen, ist für die Mittelschulstufe eine zentrale Aufgabe. Es lohnt sich, hier den Modellcharakter der Beschreibungen eines Zusammenhalts zwischen zwei Atomen immer wieder zu betonen. Dann scheint es legitim, mit Hilfe von mechanischen Analogien einen Zugang zu finden (z.B. lassen zwei Wagen mit starken Magneten auf zwei schiefen Ebenen ein stabiles System entstehen [Abb. 4]). Hier bietet die Animation 'kovalente Bindung' die Möglichkeit, die entscheidenden Grössen 'Energie', 'Energie-minimum' und 'Bindungsenergie' ins Spiel zu bringen. Die abstrakten Begriffe, die im Chemieexperiment nur indirekt fühlbar sind, lassen sich damit augenfällig demonstrieren (Abb. 5).

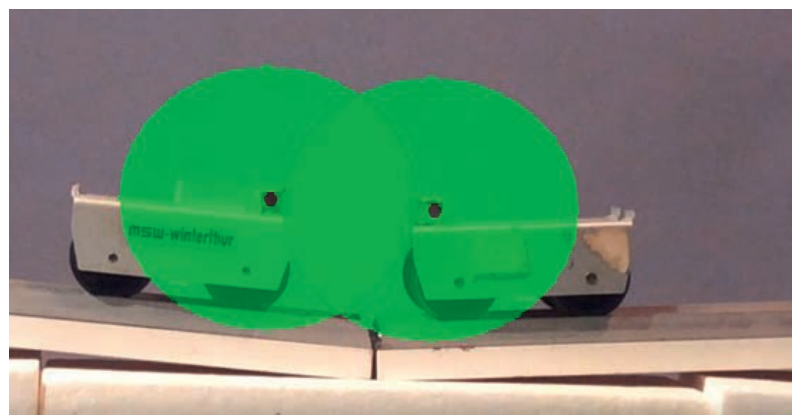

Abb. 4. Zwei kleine Wagen mit Magneten bilden auf schiefen Ebenen ein stabiles System. Sie können ergänzt werden mit zwei Kreisflächen, welche eine weitere Brücke zur Animation schlagen.

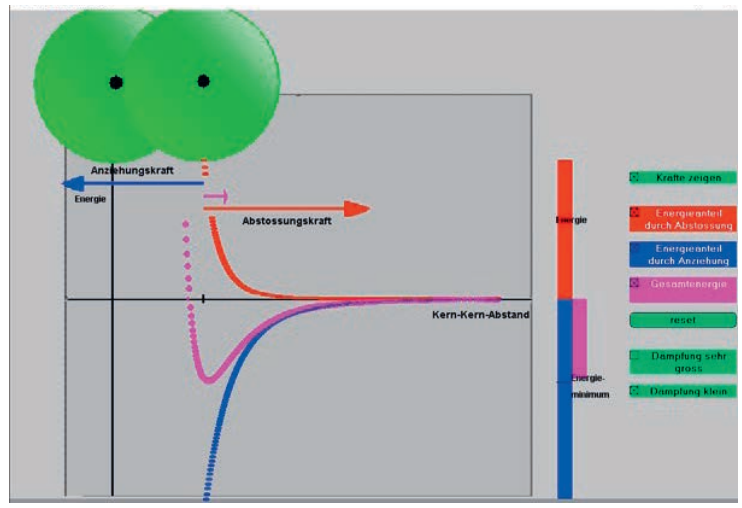

Abb. 5. Ausschnitt aus dem Programm 'kovalente Bindung'; einzelne Kräfte, Energiebeträge und deren Summen als Funktion des Kern-KernAbstandes zweier H-Atome können wahlweise zugeschaltet werden.

\section{In komplexen Vorgängen werden bekannte Einzelschritte sichtbar}

Animationen helfen, auch komplexe Vorgänge zu verstehen. Biochemische Vorgänge wie etwa enzymatische Verdauungsschritte, Photosynthese- und Proteinbiosyntheseabläufe müssen nicht mehr nur als zwar faszinierende, aber im Grunde unverständliche Wunder der Natur akzeptiert oder gar auswendig gelernt werden. In modernen Animationen, wie etwa im Programm 'Protein-biosynthese' sind die Vorgänge in Einzelschritte aufgeteilt, die die aktive Einflussnahme der Schülerinnen und Schüler verlangen. So lassen sich etwa die Schritte bei der Elongation von Peptidketten auf bekannte einfache Vorgänge reduzieren (Polarisierung von Bindungen, Partialladungen, nukleophiler Angriff, Ester- bzw. Amidbindung). Dadurch, dass die betrachtende Person aktiv Molekülteile, Liganden usw. verschieben muss und die Vorgänge beliebig oft wiederholen kann, ist der Lerneffekt gross. Zudem wird dem Gefühl, biochemische Vorgänge seien geheimnisvolle Black-Boxes, deutlich entgegengewirkt. Dank der Möglichkeit, mit Hilfe von Grafikprogrammen wie z.B. Swiss PDBviewer oder PyMOL Proteinstrukturen darzustellen, gelingt es, zugleich grafisch ansprechende und realitätsnahe Bilder zu kreieren.

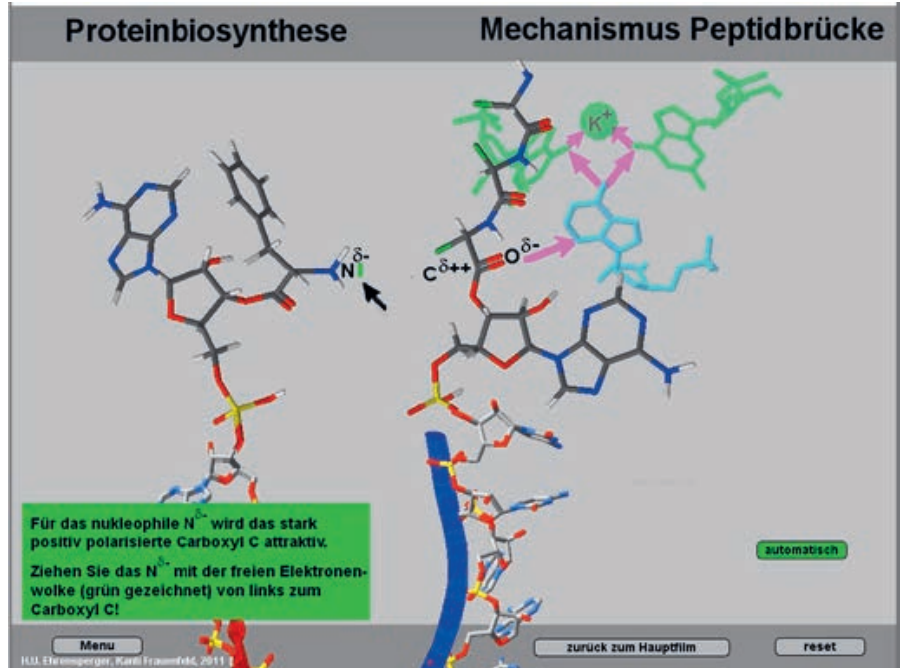

Abb. 6. Ausschnitt aus dem Programm 'Proteinbiosynthese'. Bei der Elongation wird die neue Aminosäure mit der wachsenden Proteinkette verknüpft, indem das nukleophile $\mathrm{N}$-Atom zum positiv polarisierten C-Atom verschoben werden kann. Dabei bildet sich eine neue Bindung aus.

Hinweis: Die aufgeführten Animationen können von der Website des Autors kostenfrei für PC und OSX (shockwave muss installiert sein) herunter-geladen werden.

Website: http://www.klangheimlich.ch/wp 